\title{
Contents
}

List of Contributors $\quad$ V

Preface

$\begin{array}{ll}\text { Conventions and Notations } & \text { IX }\end{array}$

I Ernst C. G. Stueckelberg: Biography and Personal Recollections

1 Overview of Stueckelberg's Life as a Scientist 3

2 Stueckelberg in Geneva and Lausanne $\quad 7$

\section{Scientific Work}

3 Stueckelberg and Molecular Physics 13

3.1 Stueckelberg's Work on Spectra of Molecules . . . . . . 14

3.2 Collisional Problems ... . . . . . . . . . . 17 


\section{Contents}

4 Stueckelberg's Covariant Perturbation Theory 25

4.1 Setting the Context:

Quantum Electrodynamics in the Early 1930s . . . . . . 26

4.2 Time-Dependent Perturbation Theory:

Problems and Issues . . . . . . . . . . . . . . . . 28

4.3 Some Remarkable Features of Stueckelberg's Scheme . . 30

4.3.1 Four-Dimensional Expansions and Contour Integrals . . . . . . . . . . . . . 31

4.3.2 The Spinor Case: Further Niceties. . . . . . . . 36

4.4 The Response to Stueckelberg's Paper . . . . . . . . . . 43

4.5 Appendix .................. . . 46

$5 \quad$ Stueckelberg's Unitary Field Theory of 1936-1939 53

5.1 From the Electric Arc to the Quantum Fields . . . . . . . 53

5.2 Covariant Quantum Electrodynamics . . . . . . . . 55

5.3 Nuclear Forces: 1930-1935 . . . . . . . . . . . . 58

5.4 The Neutrino Theory of Light . . . . . . . . . . . . . . 60

5.5 Stueckelberg's Spinor-Field World of 1936. . . . . . . . 61

5.6 Stueckelberg's Mediating Field . . . . . . . . . . . 63

5.7 Yukawa's U-Field . . . . . . . . . . . . . . . . 64

5.8 Stueckelberg's B-Field. . . . . . . . . . . . . . 66

5.9 Canonical Transformations . . . . . . . . . . . . 68

5.10 A Dream .................. . . 69

5.11 Conclusions................ . 70

6 Stueckelberg 1937-1942: The B-Field and Antiparticles as $\begin{array}{ll}\text { Time-Reversed Particles } & 73\end{array}$

6.1 Introduction. . . . . . . . . . . . . . 73

6.2 Heavy Electrons. . . . . . . . . . . . . . . 74

6.3 The Stueckelberg Mechanism and the

Stueckelberg Field. . . . . . . . . . . . . . 75 
6.4 Influence of Stueckelberg's 1938 Papers . . . . . . . . . 77

$6.5 e^{+} e^{-}$Pair Creation and Annihilation. . . . . . . . . . 80

6.6 Wave Mechanics à la Stueckelberg. . . . . . . . . . . 82

7 Stueckelberg and the S-Matrix Theory $\quad 87$

7.1 Introduction. . . . . . . . . . . . . . . 87

7.2 Causality and the S-Matrix . . . . . . . . . . . 88

7.3 Perturbative Construction of the S-Matrix . . . . . . . 92

7.4 Renormalization and Renormalization Group. . . . . . . 93

7.5 Acausality of Non-Local Interactions . . . . . . . . . 94

7.6 Concluding Remarks . . . . . . . . . . . . . . 94

7.7 Appendix: Three S-Matrix Papers . . . . . . . . . 95

7.7.1 An Unambiguous Method of Avoiding Divergence Difficulties in Quantum Theory [67]. 95

7.7.2 Causalité et Structure de la Matrice S [82]. . . . 96

7.7.3 A Propos des Divergences en Théorie des Champs Quantifiés [83]. . . . . . . . . . 97

8 Relativistic Thermodynamics $\quad 101$

8.1 Introduction. . . . . . . . . . . . . . 101

8.2 Historical Remarks . . . . . . . . . . . . . . 102

8.3 Covariant Thermodynamics: Conventional Formulation . 103

8.4 Stueckelberg-Wanders 1953 . . . . . . . . . . . . 107

8.5 Sound Speed, Causality and Stability . . . . . . . . . . 108

8.6 Stueckelberg-Wanders Rediscovered:

Phenomenological Theories of

"Divergence Tyype". . . . . . . . . . . . . . 110

\section{Stueckelberg's Selected Papers}


Relativistisch invariante Störungstheorie des Diracschen

Elektrons. I. Teil: Streustrahlung und Bremsstrahlung [20]

Austauschskräfte zwischen Elementarteilchen und Fermische Theorie des $\beta$-Zerfalls als Konsquenzen einer möglichen Feldtheorie der Materie [26]

Radioactive $\beta$-decay and nuclear exchange forces as a consequence of a unitary field theory [27]

Artificial radioactivity giving continuous $\gamma$-radiation [30]

On the existence of heavy electrons [38]

Die Wechselwirkungs Kräfte in der Elektrodynamik und in der Feldtheorie der Kernkraefte (I) [39]

Die Wechselwirkungskräfte in der Elektrodynamik und in der Feldtheorie der Kernkräfte (Teil II und III) [40]

La signification du temps propre en mécanique ondulatoire [53]

Remarque à propos de la création de paires de particules en théorie de relativité [54]

La mécanique du point matériel en théorie de relativité et en théorie des quanta [56]

An unambiguous method of avoiding divergence difficulties in quantum theory [67] 
Une propriété de l'opérateur $S$ en mécanique asymptotique [78]

A convergent expression for the magnetic moment of the neutron [81]

Causalité et structure de la Matrice $S$ [82]

A propos des divergences en théorie des champs quantifiés [83]

Relativistic Quantum Theory for Finite Time Intervals [84]

Elimination des constantes arbitraires dans la théorie relativiste des quanta [85]

Restriction of Possible Interaction in Quantum

Electrodynamics [86]

The normalization group in quantum theory [87]

Thermodynamique en Relativité Générale [89]

Stueckelberg's Complete List of Publications

Index 